
\title{
25 Research Square \\ OSI-906 Restores The Sensitivity of Ovarian Clear Cell Carcinoma To Cisplatin By Targeting The IGF1R/AKT Pathway
}

\section{Li Liu ( $\sim$ liuli93@mail.sysu.edu.cn )}

Third Affiliated Hospital of Sun Yat-Sen University https://orcid.org/0000-0002-3687-0070

\section{Changyan Liang}

Third Affiliated Hospital of Sun Yat-Sen University

Chenya Zhuo

Third Affiliated Hospital of Sun Yat-Sen University

Huiyun Jiang

Third Affiliated Hospital of Sun Yat-Sen University

\section{Huixia Ye}

Third Affiliated Hospital of Sun Yat-Sen University

\section{Tianyuan Ruan}

Third Affiliated Hospital of Sun Yat-Sen University

Jiao Song

Third Affiliated Hospital of Sun Yat-Sen University

Senwei Jiang

Third Affiliated Hospital of Sun Yat-Sen University

\section{Yu Zhang}

Third Affiliated Hospital of Sun Yat-Sen University

\section{Xiaomao Li}

Third Affiliated Hospital of Sun Yat-Sen University

\section{Research Article}

Keywords: ovarian clear cell carcinoma, IGF1, chemoresistance, proliferation, migration, apoptosis

Posted Date: June 30th, 2021

DOI: https://doi.org/10.21203/rs.3.rs-643435/v1

License: (c) (1) This work is licensed under a Creative Commons Attribution 4.0 International License. Read Full License 
Version of Record: A version of this preprint was published at Medical Oncology on January 4th, 2022. See the published version at https://doi.org/10.1007/s12032-021-01592-w. 


\section{Abstract}

Among the various histologic subtypes of ovarian cancers (OCs), ovarian clear cell carcinoma (OCCC) represents a great challenge due to its disease aggressiveness and resistance to chemotherapy. IGF1 is overexpressed in epithelial ovarian cancer (EOC), and IGF1 pathway activation is related to the chemoresistance of various cancers. In this study, we found that the expression level of IGF1 was higher in OCCC than in the most common type of OC, high-grade serous adenocarcinoma (HGSC). Then, we investigated the role of IGF1 pathway activation in the progression of OCCC, observing that activation of the IGF1 pathway using IGF1 promoted the proliferation and migration of ES2 cells, while inactivation of the IGF1 pathway using the selective IGF1R inhibitor OSI-906 reversed the alteration mediated by IGF1. Based on the role of the IGF1 pathway in cancer chemoresistance, we proposed that OSI-906 may restore the sensitivity of OCCC to cisplatin. We first validated that IGF1 increased the IC50 value of cisplatin in ES2 cells, while OSI-906 decreased it. Then we confirmed that IGF1 decreased the apoptosis rate of ES2 cells induced by cisplatin, while OSI-906 increased it. Finally, we conducted animal experiments to investigate whether OSI-906 helps cisplatin control the growth of OCCC. As expected, OSI-906 increased the effect of cisplatin in attenuating the growth of OCCC in vivo. Therefore, we conclude that using OSI906 may be an effective method to restore the sensitivity of OCCC to cisplatin by targeting the IGF1R/AKT pathway.

\section{Introduction}

Ovarian cancer $(\mathrm{OC})$ is still the most fatal gynecological malignancy, and the majority of OCs are epithelial ovarian cancer (EOC). The 5 -year-survival rate of EOC at all stages is just $47 \%{ }^{1}$. In 2020 ,it was predicted that there would be 23,900 new cases and 15,200 deaths due to ovarian cancer worldwide, and that $\mathrm{OC}$ was the seventh most common and eighth leading cause of cancer death in women ${ }^{2}$. EOC can be histologically subdivided into high grade serous cancer(HGSC), which is the main form (accounting for over $70 \%$ of EOCs), low-grade serous ovarian cancer( $10 \%$ ), ovarian clear cell carcinoma(OCCC) $₫ 5 \% \bigotimes$, endometrioid ovarian cancer $\triangle 10 \% \bigotimes$, and mucous ovarian cancer $\triangle 2.4 \% \otimes^{2-3}$. Although OCCC is not very common, its prognosis is very poor, with a 5 year survival rate of just $12.48 \%^{2}$. The median survival time of HGSOC is 40.8 months, while that of OCCC is just 21.3 months ${ }^{4}$.One of the main reasons for poor prognosis of OCCC is the high rate of resistance to standard cis-platinum and paclitaxel combination chemotherapy ${ }^{5}$. A multicenter retrospective study of patients with stage $\nabla / \nabla$ disease after surgery showed that the response rate in OCCC patients to platinum-based chemotherapy was just $11.1 \%$, compared with $72.5 \%$ in HGSOC patients ${ }^{4,6}$. Therefore, developing new methods to increase the sensitivity of OCCC to cisplatin is important to improve the outcome of OCCC patients.

Insulin-like growth factor 1(IGF1) contributes to the growth and maturation of almost every tissue. The activation of insulin-like growth factor 1 receptor (IGF1R) enhances survival, proliferation, anabolic processes, and metabolism and inhibits cell cycle arrest, apoptosis, catabolism and autography by activating the AKT pathway ${ }^{7}$. On the other side, it encourages cell survival, proliferation, RNA translation, 
and stability via activating MAPK pathway ${ }^{8}$. Abnormal activation of the IGF1 pathway contributes to the progression of various cancers. For example, IGF1 promotes colorectal cancer metastasis by mediating HOXA13-IGF1R positive feedback loop ${ }^{9}$.IGF1/IGF1R/STAT3 signaling inducible IFITM2 promotes gastric cancer growth and metastasis ${ }^{10}$.IGF1 is also significantly overexpressed in ovarian cancer ${ }^{11-12}$.IGF1 downregulates E-cadherin expression and the upregulates snail and slug, thus promoting the EMT of OC cells ${ }^{13}$.Low IGFBP-3 expression is related to advanced stage, high grade, and poor survival in OC patients ${ }^{14}$.

IGF1 also plays an important role in the chemoresistance of cancers. It was detected to be a hub gene in the chemoresistance of hepatocellular carcinoma by bioinformatic identification ${ }^{15}$. IGF1BP3 methylation deficiency in NSCLC cells treated with cisplatin may activate IGF1R/PI3K/AKT pathway, thus inducing resistance to cisplatin ${ }^{16}$. Abnormal activation of the IGF1 pathway also contributes to the resistance of OC to cisplatin ${ }^{17}$. IGF1/PI3K/NFKB/ERK signaling is overactivated in the platinum-resistant group compared with platinum-sensitive HGSC cells ${ }^{18}$. Cancer associated fibroblasts in the tumor microenvironment promote cisplatin resistance in bladder cancer cells by activating IGF1/ERß/Bcl2 signaling ${ }^{19}$. With the development of the concept of precision targeted therapy, it has become necessary to detect different molecular types of OC. A recent and unprecedented example is that OC patients with BRCA mutations will benefit more from PARP inhibitors than those without BRCA mutations ${ }^{20}$. Similarly, ARID1A deficiency increases the effect of immune checkpoint blockade therapy in $0 C^{21}$.

Here, we serendipitously observed that OCCC expressed higher levels of IGF1 than the most common subtype of HGSC in pre-experiments. Then, we validated the results in a larger number of samples. According to current reports that IGF1 pathway activation plays an important role in cancer progression, we validated that IGF1 activated the IGF1R/AKT pathway and promoted the proliferation and migration of ES2 cells, while the IGF1R inhibitor OSI-906 inactivated the IGF1R/AKT pathway and inhibited the proliferation and migration of ES2 cells. Additionally, because activation of the IGF1 pathway is related to cisplatin resistance in cancers, we validated that IGF1 treatment increased the IC50 value of cisplatin in ES2 cells to cisplatin, while OSI-906 treatment decreased it. In vivo experiments also revealed that OSI906 increased the sensitivity of ES2 cells to cisplatin. Therefore, we conclude that OSI-906 is an effective method to restore the sensitivity of OCCC to cisplatin by targeting the IGF1R/AKT pathway.

\section{Material And Methods}

\subsection{Tissue Samples and Immunochemistry Staining}

Pathological sections of HGSCs, and CCCs were purchased from Avila Biotechnology. IHC staining was performed following the protocol presented in Liu's paper ${ }^{11}$.Briefly, following deparaffinization and rehydration, 4- $\mu \mathrm{m}$-specimens underwent heat-induced epitope retrieval. Slides were blocked with $1 \%$ goat serum, and then incubated with IGF1 primary antibodies (1:125) (Affinity, Soochow, China) at $4^{\circ} \mathrm{C}$ overnight. Then囚the sections were incubated with a biotinylated goat anti-rabbit antibody (1:125) (CST, 
MA, USA) and an ABC kit was used. Slides underwent color development with DAB and hematoxylin counterstaining. Cells with cytoplasmic immunohistochemical expression were regarded as IGF1positive.The IRS system was used to evaluate the expression level of IGF1 according to the percentage of positively stained cells(no staining, score $=0 ; \otimes 10 \%$ of cells, score $=1 ; 11-50 \%$ of cells, score $=2 ; 51-80 \%$ of cells, score $=3 ; \varangle 81 \%$ of cells stained, score $=4$ ) and multiplied by staining intensity (weak staining, score $=1$; moderate staining, score $=2$; strong staining, score $=3$ ).

\subsection{ES2 cell culture}

The OCCC ES2 cell line was kindly donated by Doctor Pingping Liu from Oncology Control Center, Sun Yat-sen University, and was identified using Short Tandem Repeat analysis. ES2 cell was cultured in RPMI- 1640 medium with $10 \%$ fetal bovine serum(FBS), $100 \mathrm{U} / \mathrm{ml}$ penicillin , and $100 \mu \mathrm{g} / \mathrm{ml}$ streptomycin at $37^{\circ} \mathrm{C}$ in a humidified environment containing $5 \% \mathrm{CO} 2$.

\subsection{Western blot analysis}

Total protein of ES2 cells was extracted with RIPA lysis buffer (Beyotime, Shanghai, China) supplemented with $1 \mathrm{mM}$ PMSF and $1 \times$ phosphatase inhibitor cocktail (Beyotime, Shanghai, China). Protein quantification was conducted with BCA kits (Thermo Fisher, Rockford, IL, USA) following the manufacturer's instruction. $20 \mu \mathrm{g}$ of protein was separated on 10\% SDS-PAGE gels(EpiZyme, Shanghai, China) at constant voltage of $80 \mathrm{~V}$ in stacking gel and $120 \mathrm{~V}$ in separating gel. Then the protein was transferred onto polyvinylidene difluoride membranes at stationary electricity of $300 \mathrm{~mA}$ for $2 \mathrm{~h}$. After washed with TBST, the membranes were blocked in TBST buffer with $5 \%$ bovine serum albumin (BSA) for $2 \mathrm{~h}$ at room temperature, then incubated with the following primary antibodies at $4{ }^{\circ} \mathrm{C}$ overnight:IGF1R $\llbracket 1: 1000 \rrbracket$ monoclonal, rabbit to mouse, Abcam, Cambridge, MA,USA $\rrbracket$, phospho-IGF1R $\rrbracket 1: 1000 \rrbracket$ monoclonal, rabbit to mouse, CST, Danvers, MA,USA囚,AKT囚1:1000》monoclonal, rabbit to mouse, Abcam, Cambridge, MA,USA囚, phospho-KAT囚1:500『monoclonal, rabbit to mouse, Abcam, Cambridge, MA,USA囚, $\operatorname{GAPDH}(1: 5000$, Abways, monoclonal, rabbit to mouse, Shanghai, China).The membranes were incubated in HRP-conjugated rabbit secondary antibody (1:5000, Proteintech, Wuhan, Hubei, China) at room temperature for one hour. Finally, the protein signals were collected using enhanced chemiluminescent HRP substrate (Merck Millipore, Bedford, MA, USA).

\subsection{MTS proliferation assay}

ES2 cells were plated in 96-well plates at a density of 2,000 cells/well and cultured in $100 \mu \mathrm{l}$ of medium at $37^{\circ} \mathrm{C}$ with $5 \% \mathrm{CO} 2$.At the end of the MTS assay, each well of cells were incubated in $80 \mu \mathrm{l} \mathrm{RPMI-1640}$

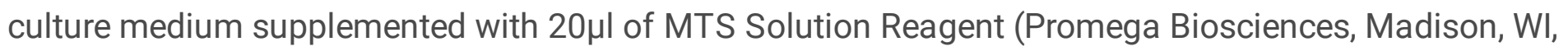
USA) was added to each well. Then, the cells were incubated at $37^{\circ} \mathrm{C}$ in a $5 \% \mathrm{CO} 2$ atmosphere for 1 hour. 
The absorbance at $490 \mathrm{~nm}$ was measured using a 96-well plate spectrometer. There were six replicates in each group.

\subsection{Wound healing assay}

Cell motility was evaluated by a wound-healing assay. Initially, $10^{5}$ cells were plated in six-well plates and cultured overnight. The cells were mechanically scratched with pipette tips and washed three times using

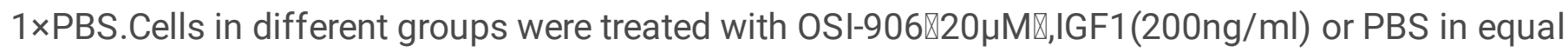
volumes. After 16 hours of incubation, the cells that had migrated to the wound surface were recorded under an inverted microscope. The wound width was measured using ImageJ software. The assays were performed in triplicate and repeated at least three times.

\subsection{Flow cytometry}

ES2 cells exposed to IGF1, OSI-906 or cisplatin for 48 hours were harvested and incubated with $\mathrm{PI}$ and Annexin V antibodies (Key Gene Bio Tech, Nanjing, China) at room temperature for 30 minutes. Then, the cells were washed twice. Apoptosis was determined using a flow cytometer. The results were analyzed using Flow Jo software.

\subsection{Animal experiment}

All animal experiments received approval from the Institutional Use and Care of Animal Committee of The Third Affiliated Hospital, Sun Yat-sen University. BALB/c nude mice were purchased from Hunan Silaike Jingda Laboratory Animal Co., Ltd. ES2 cells ( $3 \times 10^{6}$ cells in $200 \mu$ l of PBS) were intraperitoneally inoculated into nude mice to generate an abdominal ovarian cancer model. Two weeks later, animals were divided into control, OSI-906, cisplatin and combination groups. Animals in the OSI-906 group were intraperitoneally injected with OSI-906 $(25 \mathrm{mg} / \mathrm{kg})$ twice a week, those in the cisplatin group were intraperitoneally injected with cisplatin $(3 \mathrm{mg} / \mathrm{kg}$ ) twice a week, those in the combination group were intraperitoneally injected with both medicines, and those in the control group were treated with PBS. The treatment in each group all lasted two weeks. All animals were euthanized by $\mathrm{CO} 2$ asphyxiation with subsequent cervical dislocation two weeks after the treatment. The tumors were isolated by dissection and weighed using an electronic balance. Tumor tissues were stained by HE.

\subsection{Hematoxylin-eosin (HE) staining}

The paraffin-embedded sections were dewaxed. Then the nuclei were stained using hematoxylin for 5 minutes. The cells were then dissimilated with $1 \%$ ethanol-hydrochloric acid for $30 \mathrm{~s}$, and the cytoplasm was stained using eosin for 2 minutes. The sections were sealed using neutral gum. Finally, images were captured under an optical microscope. 


\subsection{Statistical analysis}

All experiments in this study were independently repeated at least three times. SPSS 21.0 statistical software was used for data analysis. The measurement data are expressed as the mean \pm standard error

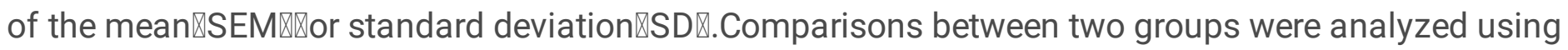
Student's t-test or the Mann-Whitney test. $\mathrm{P} \otimes 0.05$ was considered to be statistically significant.

\section{Results}

\subsection{IGF1 expression is abnormally higher in OCCC tissues}

IGF1 is highly expressed in ovarian cancer ${ }^{11}$,but whether different pathological types of OC express different levels of IGF1 has not been reported. We first noticed that the IGF1 level of CCC was significantly higher than that of HGSC tissues. To validate this, we expended samples from 10 ovarian benign carcinoma, 10 HGSC and 10 CCC.As predicted, CCC tissues expressed higher levels of IGF1 than HGSC tissues(Fig. 1A).We semi-quantitatively evaluated the IGF1 level using the IRS system, and found that score of the IGF1 expression level was higher in CCC than in HGSC (Fig. 1B).

\subsection{IGF1 promotes the proliferation and migration of OCCC cells}

IGF1 pathway activation is closely related to the proliferation and migration of cancer cells. To investigate the role of IGF1 in the promotion of OCCC, we used IGF1 to treat OCCC cells to activate the IGF1R/AKT pathway (Fig. 2A). We evaluated the effect of IGF1 on the proliferation of OCCC cells. Both the gross appearance (Fig. 2B) and the MTS assay (Fig. 2C) showed that IGF1 promoted the proliferation of OCCC cells. At the same time, we evaluated the effect of IGF1 on the migration ability of OCCC cells, and the results showed that IGF1 accelerated their migration of OCCC cells (Fig. 2D).

\subsection{Targeting IGF1R using OSI-906 reverses the proliferation and migration of OCCC cells induced by IGF1}

Based on these results, we proposed that targeting the IGF1 pathway could attenuate the proliferation and migration of OCCC. Actually, in our another report, we found that OSI-906 could reverse the proliferation and migration of $\mathrm{OC}$ cells induced by tumor associated macrophages ${ }^{11}$. Here, we tried to use OSI-906 to inactivate the IGF1 pathway in OCCC cells. We observed that OSI-906 successfully inhibited the activation of the IGF1 pathway in OCCC cells treated with IGF1 (Fig. 3A). Under a light microscope, we observed that OSI-906 successfully inhibited the proliferation of ES2 cells treated with IGF1(Fig. 3B).At the same time, we conducted an MTS assay to evaluate the effect of OSI-906 on the proliferation of ES2 cells, and observed that proliferation induced by IGF1 was negatively reversed(Fig. 3C). Finally, we 


\subsection{OSI-906 restores the sensitivity of OCCC to cisplatin in vitro}

It has been reported that activation of the IGF1 pathway contributes to platinum resistance in many cancers such as lung cancer and hepatocellular carcinoma ${ }^{15,22}$. Thus, we investigated its role in cisplatin resistance in OCCC. We observed that IGF1 treatment increased the IC50 value of cisplatin in OCCC cells, while OSI-906 treatment decreased it(Fig. 4A and B).Furthermore, we investigated the effects of IGF1 and OSI-906 on the apoptosis of OCCC cells induced by cisplatin, and the results showed that IGF1 treatment reduced the apoptosis induced by cisplatin, while OSI-906 had the opposite function(Fig. $4 C$ and $D)$.

\subsection{Targeting IGF1R restores cisplatin the sensitivity of OCCC in vivo}

To validate the in vitro results, we conducted animal experiments. Four-to-six-week-old mice were equally divided into a control group, a cisplatin group, an OSI-906 group and a combination group.ES2 cells were injected into abdominal cavity of BALB/c nude mice to generate an OCCC tumor model. Two weeks later, the BALB/c nude mice with tumors received different treatments. Two weeks after the treatments were terminated, the mice were sacrificed, and tumors in the abdominal cavity were recorded and weighed (Fig. 5A). Obviously, we observed that cisplatin significantly restored the sensitivity of OCCC to cisplatin (Fig. 5B). The tumor weight was significantly lower in the combination group than the other groups (Fig. 5C). Finally, the tumors were analyzed using HE staining (Fig. 5D).

\section{Discussion}

Chemoresistance is one of the most significant challenges affecting the prognosis of OCCC. As summarized in the schematic diagram, based on the initial result that the expression level of IGF1 was significantly higher in OCCC tissues than in HGSC tissues(the most common type of ovarian cancer), we validated that IGF1/IGF1R/AKT signaling activation contributed to malignant behaviors including the proliferation and migration of OCCC cells, while blocking the IGF1 pathway using OSI-906 reversed these effects. Then, we observed that OSI-906 restored the sensitivity of OCCC cells to cisplatin. Finally, we verified these results in vivo.

Different types of ovarian cancers have different molecular profiles. For example, hypoxia-inducible factor 1 alpha(HIF1a) is overexpressed in CCC compared with other types of ovarian cancer ${ }^{23}$. The concept of precision targeted therapy calls for detecting genes that are expressed at different levels in 
different types of ovarian cancers, and great progress has been gained in precision therapy in recent years. A representative breakthrough is that the SOLO1 phase 3 clinical trial showed that maintenance therapy with the poly ADP-ribose polymerase inhibitor olaparib resulted in a 70\% lower risk of disease progression or death in newly diagnosed advanced ovarian cancer patients with BRCA1/2 mutations ${ }^{24}$.Additionally, targeting the VEGF/VEGFR pathway has also shown great potential in the treatment of $\mathrm{OC}$ with a great effect on inhibiting angiogenesis ${ }^{25}$.In addition, many potential therapy targets against specific molecular alterations have been investigated for the treatment of OC, including BRCA1/2 mutations, the PI3K/AKT/mTOR pathway, TP53 mutation, the RAS/RAF/MEK/ERK pathway, and HER2 status. Our previously published study revealed that IGF1 levels are higher in EOC than in benign ovarian carcinoma. In this study, we found that OCCC tissues expressed higher levels of IGF1 than HGSC tissues(Fig. 1), which suggested that the IGF1 pathway may play an important role in the progression of OCCC, and targeting the IGF1 pathway could be effective in controlling OCCC, which is supported by Nagle's conclusion that the loss of E-cadherin enhances IGF1 pathway activation and sensitizes breast cancers to IGF1R/INsR inhibitors ${ }^{26}$

IGF1 pathway activation promotes the proliferation, survival and metabolism of cells ${ }^{17,27}$. IGF1 pro-forms can induce breast cancer cell proliferation via the IGF1 receptor ${ }^{28}$.IGF1 pathway activation is also related to the migration of hepatocellular carcinoma ${ }^{29}$. Here, we observed that after activating the IGF1R/AKT pathway using IGF1, the proliferation and migration of OCCC cells were promoted (Fig. 2), while the proliferation and migration of OCCC cells enhanced by IGF1 were attenuated when the IGF1 pathway was blocked by the IGF1R inhibitor OSI-906(Fig. 3). There have been some evidence that targeting the IGF1 pathway may be effective in controlling a variety of cancers. An IGF1 inhibitor could strongly control cell growth of ER positive breast cancer cells ${ }^{30}$.Targeting IGF1R amplifies the effects of chemotherapy via autophagy and immune-dependent mechanisms in triple-negative breast cancer ${ }^{31}$. Some researchers have developed IGF1R targeted theranostic nanoparticles for precision therapy of pancreatic cancer ${ }^{32}$.However, there is still a lack of relevant researches in OC, especially in OCCC.

The IGF1 pathway has also been reported to be related to chemoresistance in some types of cancers, which we reviewed in our previous paper ${ }^{33}$. It has been proven that chemotherapy-induced IGF1R activation provides for enhanced sensitivity to IGF1R targeted therapy ${ }^{34}$. Some researchers have reported that chemoresistance in pancreatic cancer is driven by stroma derived IGFs ${ }^{35}$.IGF1 mediates 5fluorouracil chemoresistance in esophageal carcinoma cells by increasing survival stability ${ }^{36}$. There have been some reports that targeting IGF1 can reverse chemotherapy in cancers. Inhibition of IGF1R can overcome the chemoresistance induced by IGFBP7 in T- ALL ${ }^{37}$.IGF1R axis inhibition restores the dendritic cell antitumor response in ovarian cancer ${ }^{38}$.In this study, we observed that IGF1 treatment increased the IC50 value of cisplatin in OCCC cells, while OSI-906 reversed it. We observed the effects of IGF1 and OSI906 on the apoptosis of ES2 cells induced by cisplatin, and found that OSI-906 restored the sensitivity of OCCC to cisplatin in vitro and in vivo (Figs. 4 and 5). These results suggested that targeting the IGF1 pathway has the potential to reverse the resistance of OCCC to cisplatin. 
In conclusion, our study suggested that abnormally high levels of IGF1 may play an important role in the chemoresistance of OCCC, and that OSI-906 can effectively restore the sensitivity of ovarian clear cell carcinoma to cisplatin by targeting the IGF1R/AKT pathway. In the future:(1) We will investigate the toxicity of OSI-906 to each organ of mice; (2)We will research the mechanism of IGF1 overexpression in OCCC; (3) A clinical study will be necessary to compare whether OCCC patients will benefit more than HGSC patients from therapy targeting the IGF1R pathway (4) As noncoding RNAs have been found to be closely related to the regulation of downstream genes, in the future, we will investigate ncRNAs that may participate in the regulation of IGF1s. Noncoding RNAs such as microRNAs, IncRNAs, and circRNAs have also been considered for use the targeted therapy of ovarian cancer. (5) Furthermore, the tumor microenvironment or chemotherapy may contribute to the upregulation of IGF1 in cancer cells. For example, radiotherapy-activated cancer associated fibroblasts promote tumor progression through paracrine IGF1R activation ${ }^{39}$.More research needs to be conducted to deeply explore the mechanism by which IGF1 is overexpressed in OCCC.

\section{Declarations}

\section{Acknowledgments}

Thank Tianyun Lan, Baoling Chen, Haibo Zhang from central laboratory of The Third Affiliated Hospital of Sun Yat-sen University for their technology instructions. Thank Pingping Liu from Oncology Control Center, Sun Yat-sen University for kindly donating ES2 cells.

\section{Declarations of Compliance with Ethical Standards}

Funding This study was financially supported by grants from China Postdoctoral Science Foundation(2020M683101).

Conflicts of interest The authors declare that they have no competing interests.

Availability of data and material Data and materials in the current study are available from the corresponding author on reasonable request.

Code availability Not applicable.

Authors' contributions Li Liu and Changyan Liang designed the study and conducted molecular biology experiments and wrote the manuscript. Tianyuan Ruan, Jiao Song conducted animal experiments. Chenya Zhuo ,Huiyun Jiang and Senwei Jiang were responsible for data analyze, literature research and update. Yu Zhang and Xiaomao Li designed and guided the study, and revised the manuscript.

Ethics approval The studies involving human participants were reviewed and approved by Committee on Medical Ethics of The Third Affiliated Hospital, Sun Yat-sen University. The study complied with the ethical standards of the committee responsible for human experimentation (institutional and national), and with the Helsinki Declaration of 1975, as revised in 2013.Animal experiment procedures in this study 
were reviewed and approved by Care of Animal Committee of Sun Yat-sen University. The study complied with the US National Research Council's "Guide for the Care and Use of Laboratory Animals".

Informed consent Patients who donated clinical samples were fully informed of the usage of the samples as well as the study protocol prior to sample collection. They provided written informed consents.

Consent for publication Written informed consent for publication was obtained from all participants.

\section{References}

1. Torre LA, Trabert B, DeSantis CE, Miller KD, Samimi G, Runowicz CD, Gaudet MM, Jemal A, Siegel RL. Ovarian cancer statistics, 2018. CA Cancer J Clin. 2018;68(4):284-96. https://doi.org/10.3322/caac.21456.

2. Lheureux S, Braunstein M, Oza AM. Epithelial ovarian cancer: Evolution of management in the era of precision medicine. CA Cancer J Clin. 2019;69(4):280-304. https://doi.org/10.3322/caac.21559.

3. Rojas V, Hirshfield KM, Ganesan S, Rodriguez-Rodriguez L. Molecular Characterization of Epithelial Ovarian Cancer: Implications for Diagnosis and Treatment. Int J Mol Sci. 2016;17:(12).

4. Zhou G, Zhu Z, Li L, Ding J. Resibufogenin inhibits ovarian clear cell carcinoma (OCCC) growth, and migration of OCCC cells, by down-regulating the PI3K/AKT and actin cytoskeleton signaling pathways. Am J Transl Res. 2019;11(10):6290-303.

5. Okamoto A. Management and new strategy of ovarian clear cell carcinoma. Int J Clin Oncol. 2020;25(3):418. https://doi.org/10.1007/s10147-020-01626-9.

6. Sugiyama T, Kamura T, Kigawa J, Terakawa N, Kikuchi Y, Kita T, Suzuki M, Sato I, Taguchi K. Clinical characteristics of clear cell carcinoma of the ovary: a distinct histologic type with poor prognosis and resistance to platinum-based chemotherapy. Cancer. 2000;88(11):2584-9.

7. Manning BD, Toker A, Signaling AKT/PKB. Navigating the Network. Cell. 2017;169(3):381-405. https://doi.org/10.1016/j.cell.2017.04.001.

8. Roux PP, Blenis J. ERK and p38 MAPK-activated protein kinases: a family of protein kinases with diverse biological functions. Microbiol Mol Biol Rev. 2004;68(2):320-44.

9. Qiao C, Huang W, Chen J, Feng W, Zhang T, Wang Y, Liu D, Ji X, Xie M, Sun M, Fan D, Wu K, Xia L. IGF1-mediated HOXA13 overexpression promotes colorectal cancer metastasis through upregulating ACLY and IGF1R. Cell Death Dis. 2021;12(6):564. https://doi.org/10.1038/s41419-021-03833-2.

10. Xu L, Zhou R, Yuan L, Wang S, Li X, Ma H, Zhou M, Pan C, Zhang J, Huang N, Shi M, Bin J, Liao Y, Liao W. IGF1/IGF1R/STAT3 signaling-inducible IFITM2 promotes gastric cancer growth and metastasis. Cancer Lett 2017, 393, 76-85. https://doi.org/10.1016/j.canlet.2017.02.014.

11. Liu L, Wang X, Li X, Wu X, Tang M, Wang X. Upregulation of IGF1 by tumor-associated macrophages promotes the proliferation and migration of epithelial ovarian cancer cells. Oncol Rep 2018, 39 (2), 818-826. https://doi.org/10.3892/or.2017.6148. 
12. Rohr I, Zeillinger R, Heinrich M, Concin N, Vergote I, Nassir M, Mahner S, Van Nieuwenhuysen E, Trillsch F, Cacsire-Tong D, Chekerov R, Sehouli J, Braicu El. Role of IGF-I in Primary Ovarian Cancer - A Study of the OVCAD European Consortium. Anticancer Res. 2016;36(3):1015-22.

13. Lau M-T, Leung PCK. The PI3K/Akt/mTOR signaling pathway mediates insulin-like growth factor 1induced E-cadherin down-regulation and cell proliferation in ovarian cancer cells. Cancer Lett 2012, 326 (2), 191-198. https://doi.org/10.1016/j.canlet.2012.08.016.

14. Torng PL, Lee YC, Huang CY, Ye JH, Lin YS, Chu YW, Huang SC, Cohen P, Wu CW, Lin CT. Insulin-like growth factor binding protein-3 (IGFBP-3) acts as an invasion-metastasis suppressor in ovarian endometrioid carcinoma. Oncogene. 2008;27(15):2137-47.

15. Huang DJ, Huang JZ, Yang J, Li YH, Luo YC, He HY, Huang HJ. Bioinformatic identification of IGF1 as a hub gene in hepatocellular carcinoma (HCC) and in-vitro analysis of the chemosensitizing effect of miR-379 via suppressing the IGF1/IGF1R signaling pathway. Eur Rev Med Pharmacol Sci. 2016;20(24):5098-106.

16. Cortés-Sempere M, de Miguel MP, Pernía O, Rodriguez C, de Castro Carpeño J, Nistal M, Conde E, López-Ríos F, Belda-Iniesta C, Perona R, Ibanez, de Caceres. I., IGFBP-3 methylation-derived deficiency mediates the resistance to cisplatin through the activation of the IGFIR/Akt pathway in non-small cell lung cancer. Oncogene 2013, 32 (10), 1274-1283. https://doi.org/10.1038/onc.2012.146.

17. Liu L, Li X. A Review of IGF1 Signaling and IGF1-related Long Noncoding RNAs in Chemoresistance of Cancer. Curr Cancer Drug Targets 2020, 20 (5), 325-334. https://doi.org/10.2174/1568009620666200228123754.

18. Eckstein N, Servan K, Hildebrandt B, Pölitz A, von Jonquières G, Wolf-Kümmeth S, Napierski I, Hamacher A, Kassack MU, Budczies J, Beier M, Dietel M, Royer-Pokora B, Denkert C, Royer H-D. Hyperactivation of the insulin-like growth factor receptor I signaling pathway is an essential event for cisplatin resistance of ovarian cancer cells. Cancer research 2009, 69 (7), 2996-3003. https://doi.org/10.1158/0008-5472.CAN-08-3153.

19. Long X, Xiong W, Zeng X, Qi L, Cai Y, Mo M, Jiang H, Zhu B, Chen Z, Li Y. Cancer-associated fibroblasts promote cisplatin resistance in bladder cancer cells by increasing IGF-1/ER $/ \mathrm{Bcl}-2$ signalling. Cell Death Dis. 2019;10(5):375. https://doi.org/10.1038/s41419-019-1581-6.

20. Oza AM, Cibula D, Benzaquen AO, Poole C, Mathijssen RHJ, Sonke GS, Colombo N, Špaček J, Vuylsteke P, Hirte H, Mahner S, Plante M, Schmalfeldt B, Mackay H, Rowbottom J, Lowe ES, Dougherty B, Barrett JC, Friedlander M. Olaparib combined with chemotherapy for recurrent platinumsensitive ovarian cancer: a randomised phase 2 trial. Lancet Oncol 2015, 16 (1), 87-97. https://doi.org/10.1016/S1470-2045(14)71135-0.

21. Shen J, Ju Z, Zhao W, Wang L, Peng Y, Ge Z, Nagel ZD, Zou J, Wang C, Kapoor P, Ma X, Ma D, Liang J, Song S, Liu J, Samson LD, Ajani JA, Li G-M, Liang H, Shen X, Mills GB, Peng G. ARID1A deficiency promotes mutability and potentiates therapeutic antitumor immunity unleashed by immune checkpoint blockade. Nat Med 2018, 24 (5), 556-562. https://doi.org/10.1038/s41591-018-0012-z. 
22. Sun Y, Zheng S, Torossian A, Speirs CK, Schleicher S, Giacalone NJ, Carbone DP, Zhao Z, Lu B. Role of insulin-like growth factor-1 signaling pathway in cisplatin-resistant lung cancer cells. Int $J$ Radiat Oncol Biol Phys 2012, 82 (3), e563-e572. https://doi.org/10.1016/j.jijobp.2011.06.1999.

23. Lee S, Garner EIO, Welch WR, Berkowitz RS, Mok SC. Over-expression of hypoxia-inducible factor 1 alpha in ovarian clear cell carcinoma. Gynecol Oncol. 2007;106(2):311-7.

24. Moore K, Colombo N, Scambia G, Kim B-G, Oaknin A, Friedlander M, Lisyanskaya A, Floquet A, Leary A, Sonke GS, Gourley C, Banerjee S, Oza A, González-Martín A, Aghajanian C, Bradley W, Mathews C, Liu J, Lowe ES, Bloomfield R, DiSilvestro P. Maintenance Olaparib in Patients with Newly Diagnosed Advanced Ovarian Cancer. N Engl J Med. 2018;379(26):2495-505. https://doi.org/10.1056/NEJMoa1810858.

25. Lan C-Y, Wang Y, Xiong Y, Li J-D, Shen J-X, Li Y-F, Zheng M, Zhang Y-N, Feng Y-L, Liu Q, Huang H-Q, Huang X. Apatinib combined with oral etoposide in patients with platinum-resistant or platinumrefractory ovarian cancer (AEROC): a phase 2, single-arm, prospective study. Lancet Oncol. 2018;19(9):1239-46. https://doi.org/10.1016/S1470-2045(18)30349-8.

26. Nagle AM, Levine KM, Tasdemir N, Scott JA, Burlbaugh K, Kehm J, Katz TA, Boone DN, Jacobsen BM, Atkinson JM, Oesterreich S, Lee AV. Loss of E-cadherin Enhances IGF1-IGF1R Pathway Activation and Sensitizes Breast Cancers to Anti-IGF1R/InsR Inhibitors. Clin Cancer Res. 2018;24(20):5165-77. https://doi.org/10.1158/1078-0432.CCR-18-0279.

27. Hakuno F, Takahashi S-I. IGF1 receptor signaling pathways. J Mol Endocrinol. 2018;61(1):T69-86. https://doi.org/10.1530/JME-17-0311.

28. De Santi M, Annibalini G, Barbieri E, Villarini A, Vallorani L, Contarelli S, Berrino F, Stocchi V, Brandi G. Human IGF1 pro-forms induce breast cancer cell proliferation via the IGF1 receptor. Cell Oncol (Dordr). 2016;39(2):149-59. https://doi.org/10.1007/s13402-015-0263-3.

29. D'Alessandro R, Refolo MG, Lippolis C, Carella N, Messa C, Cavallini A, Carr BI. Strong enhancement by IGF1-R antagonists of hepatocellular carcinoma cell migration inhibition by Sorafenib and/or vitamin K1. Cell Oncol (Dordr). 2018;41(3):283-96. https://doi.org/10.1007/s13402-018-0370-z.

30. Iida M, Tsuboi K, Niwa T, Ishida T, Hayashi S-I. Compensatory role of insulin-like growth factor 1 receptor in estrogen receptor signaling pathway and possible therapeutic target for hormone therapyresistant breast cancer. Breast Cancer 2019, 26 (3), 272-281. https://doi.org/10.1007/s12282-0180922-0.

31. Wu Q, Tian A-L, Li B, Leduc M, Forveille S, Hamley P, Galloway W, Xie W, Liu P, Zhao L, Zhang S, Hui P, Madeo F, Tu Y, Kepp O, Kroemer G. IGF1 receptor inhibition amplifies the effects of cancer drugs by autophagy and immune-dependent mechanisms. J Immunother Cancer 2021, 9 (6). https://doi.org/10.1136/jitc-2021-002722.

32. Zhou H, Qian W, Uckun FM, Wang L, Wang YA, Chen H, Kooby D, Yu Q, Lipowska M, Staley CA, Mao H, Yang L. IGF1 Receptor Targeted Theranostic Nanoparticles for Targeted and Image-Guided Therapy of Pancreatic Cancer. ACS Nano. 2015;9(8):7976-91. https://doi.org/10.1021/acsnano.5b01288. 
33. Liu L, Li X. A review of IGF1 signaling and IGF1-related long noncoding RNAs in chemoresistance of cancer. Curr Cancer Drug Targets. 2020. https://doi.org/10.2174/1568009620666200228123754.

34. Dallas NA, Xia L, Fan F, Gray MJ, Gaur P, van Buren G, Samuel S, Kim MP, Lim SJ, Ellis LM. Chemoresistant colorectal cancer cells, the cancer stem cell phenotype, and increased sensitivity to insulin-like growth factor-I receptor inhibition. Cancer research. 2009;69(5):1951-7. https://doi.org/10.1158/0008-5472.CAN-08-2023.

35. Ireland L, Santos A, Ahmed MS, Rainer C, Nielsen SR, Quaranta V, Weyer-Czernilofsky U, Engle DD, Perez-Mancera PA, Coupland SE, Taktak A, Bogenrieder T, Tuveson DA, Campbell F, Schmid MC, Mielgo A. Chemoresistance in Pancreatic Cancer Is Driven by Stroma-Derived Insulin-Like Growth Factors. Cancer research. 2016;76(23):6851-63. https://doi.org/10.1158/0008-5472.CAN-16-1201.

36. Juan H-C, Tsai H-T, Chang P-H, Huang C-YF, Hu C-P, Wong F-H. Insulin-like growth factor 1 mediates 5fluorouracil chemoresistance in esophageal carcinoma cells through increasing survivin stability. Apoptosis 2011, 16 (2), 174-183. https://doi.org/10.1007/s10495-010-0555-z.

37. Bartram I, Erben U, Ortiz-Tanchez J, Blunert K, Schlee C, Neumann M, Heesch S, Baldus CD. Inhibition of IGF1-R overcomes IGFBP7-induced chemotherapy resistance in T-ALL. BMC Cancer. 2015;15:663. https://doi.org/10.1186/s12885-015-1677-z.

38. Somri-Gannam L, Meisel-Sharon S, Hantisteanu S, Groisman G, Limonad O, Hallak M, Bruchim I. IGF1R Axis Inhibition Restores Dendritic Cell Antitumor Response in Ovarian Cancer. Trans/ Oncol 2020, 13 (8), 100790. https://doi.org/10.1016/j.tranon.2020.100790.

39. Tommelein J, De Vlieghere E, Verset L, Melsens E, Leenders J, Descamps B, Debucquoy A, Vanhove C, Pauwels P, Gespach CP, Vral A, De Boeck A, Haustermans K, de Tullio P, Ceelen W, Demetter P, Boterberg T, Bracke M, De Wever O. Radiotherapy-Activated Cancer-Associated Fibroblasts Promote Tumor Progression through Paracrine IGF1R Activation. Cancer research. 2018;78(3):659-70. https://doi.org/10.1158/0008-5472.CAN-17-0524.

\section{Figures}


A

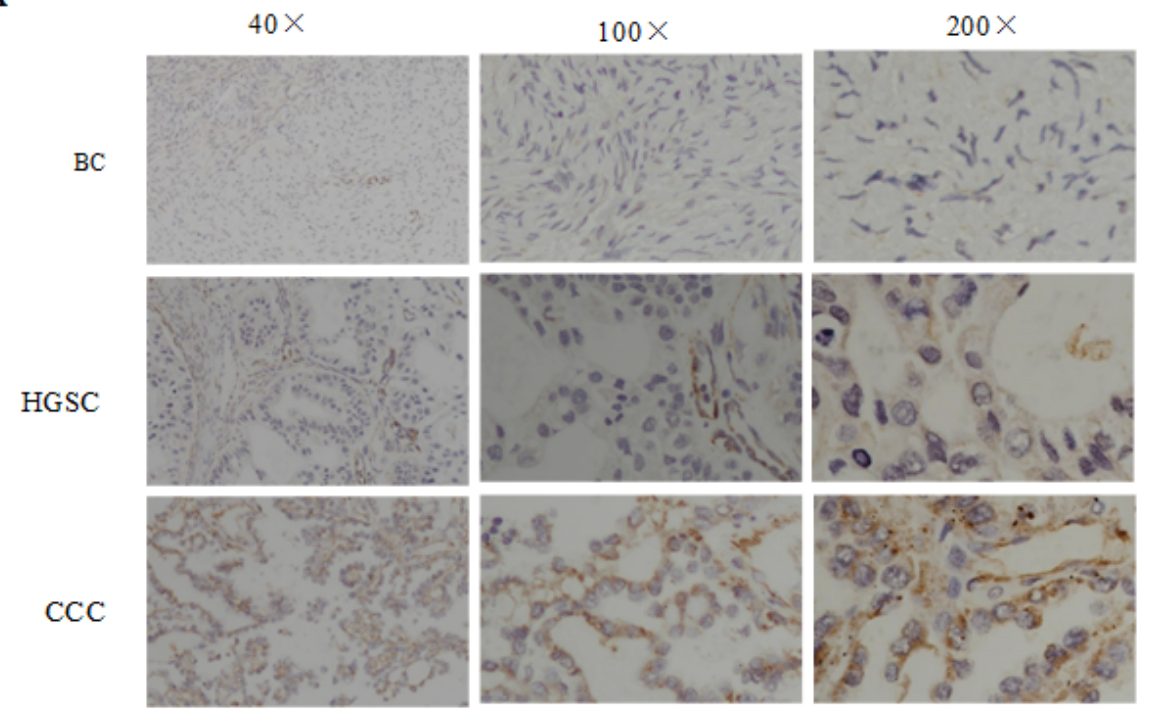

B

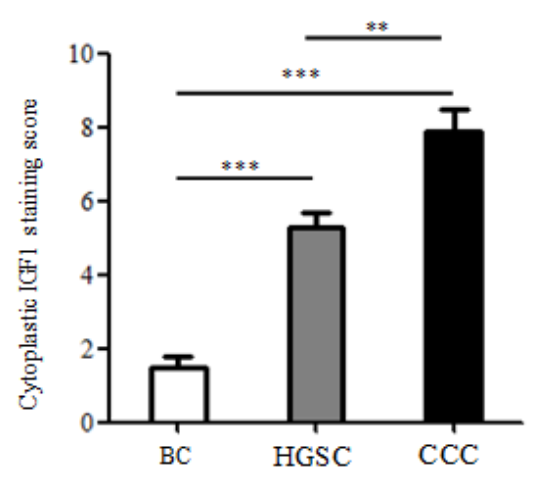

Figure 1

IGF1 expression is higher in OCCC than HGSC cells. (A)IHC staining showed that CCCs express higher IGF1 than ovarian benign carcinomas and HGSCs. (B)The IRS score of OCCC tissues was higher than that of HGSC tissues. ${ }^{\star *} p \otimes 0.01,{ }^{* \star *} p \otimes 0.001$.

A
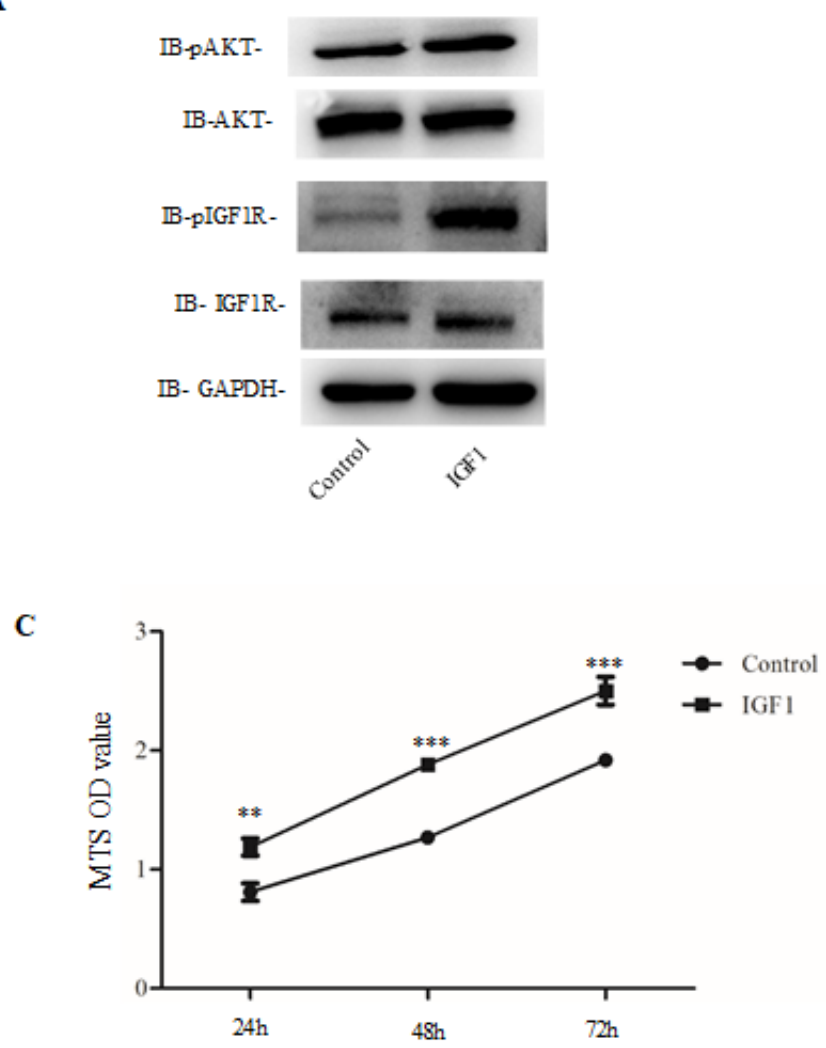

$40 \times$

$100 \times$

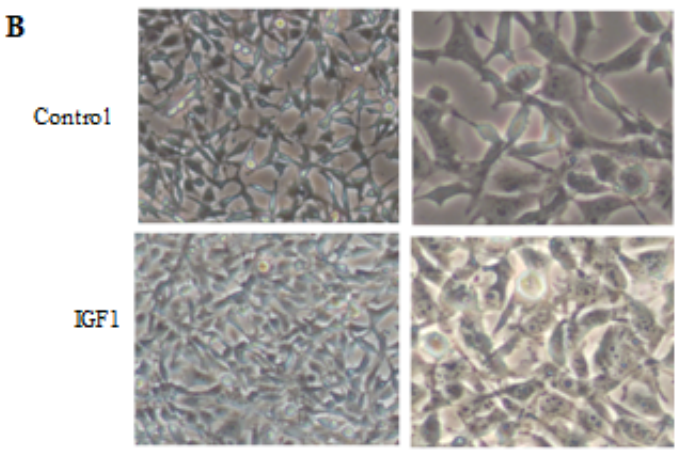

D
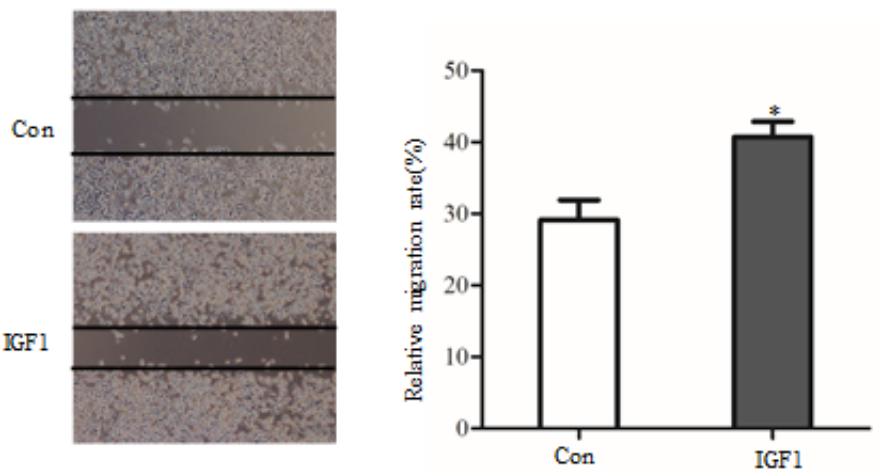

Figure 2 
IGF1 pathway activation accelerates the proliferation and migration of OCCC. (A)Western blotting was used to determine the activation of IGF1/AKT pathway in ES2 cells treated with IGF1. (B, C) Light microscopy and MTS assay were used to evaluate the effect of IGF1区200ng/m/囚on the proliferation of ES2 cells. (D)The migration ability of ES2 cells was measured by wound healing assays after exposure to

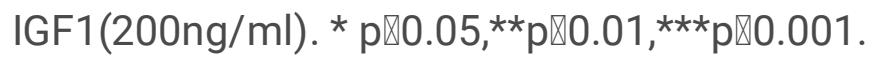

A

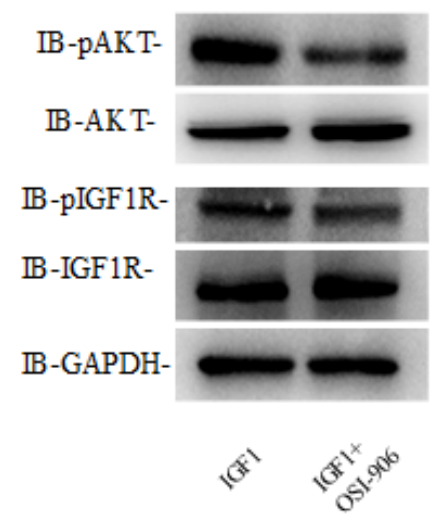

C

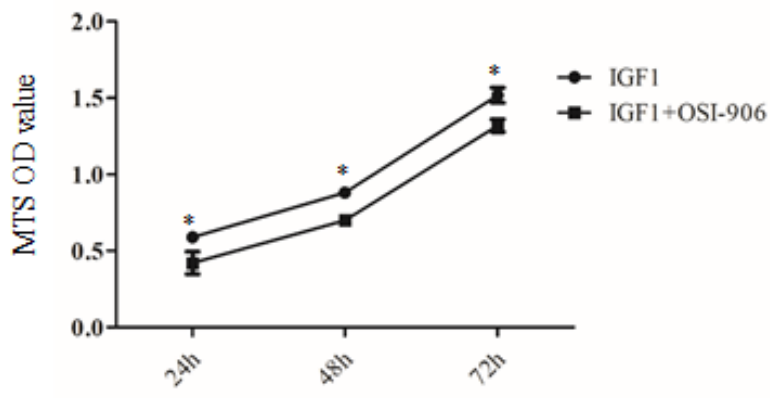

B

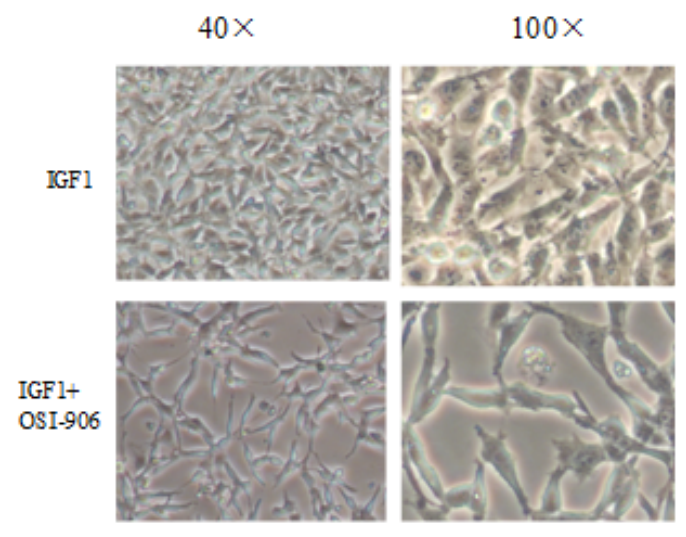

D

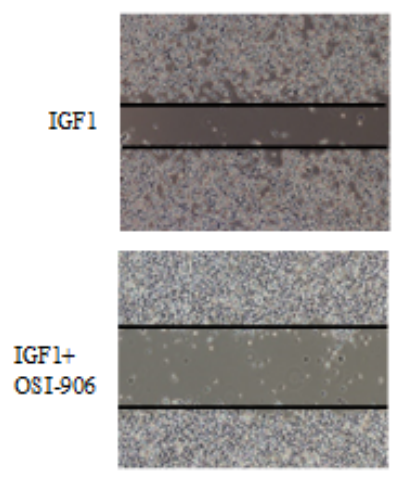

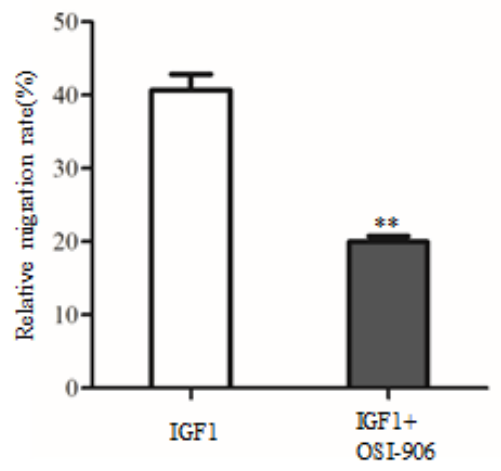

\section{Figure 3}

IGF1 pathway inactivation reversed the proliferation and migration of OCCC cells induced by IGF1. (A)Western blotting was used to evaluate the effect of OSI-906 on the activation of the IGF1R/AKT pathway in ES2 cells treated with IGF1. (B)Representative appearance of ES2 cells exposed to IGF1 under a microscope after treated with OSI-906. (C)The proliferation ability of ES2 cells exposed to IGF1 was evaluated by MTS assay after treated with OSI-906. (D)The migration ability of ES2 cells exposed to IGF1 was measured by wound healing assays after treated with OSI-906.*pष0.05, ${ }^{\star \star} \mathrm{p} \otimes 0.01$. 
A

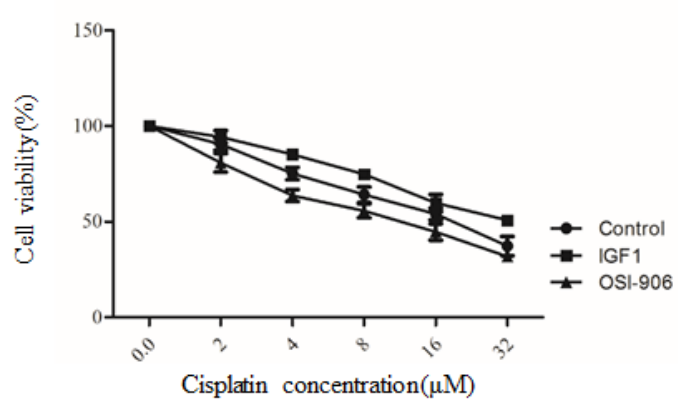

C

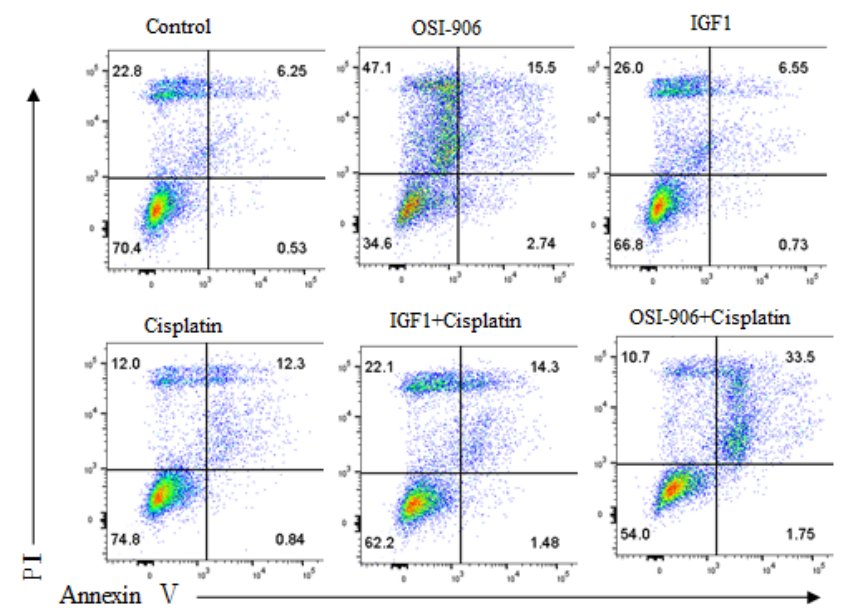

B

\begin{tabular}{|l|l|}
\hline Group & IC50 values $(\boldsymbol{\mu M})$ \\
\hline Control & 18.76 \\
\hline IGF1 & 29.59 \\
\hline OSI-906 & 11.09 \\
\hline
\end{tabular}

D

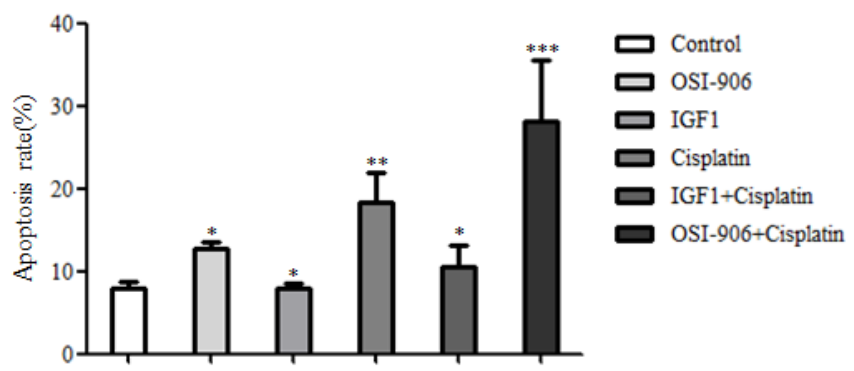

\section{Figure 4}

OSI-906 restores the sensitivity of OCCC to cisplatin in vitro. (A and B) The IC50 values of ES2 cells exposed to IGF1 and OSI-906 was measured by MTS assay. ( $C$ and D) The apoptosis of ES2 exposed to

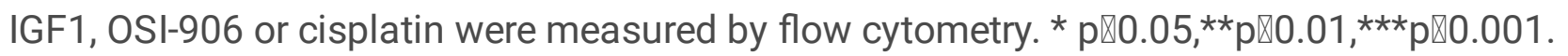

A

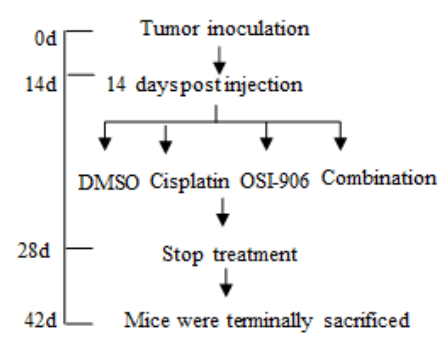

C

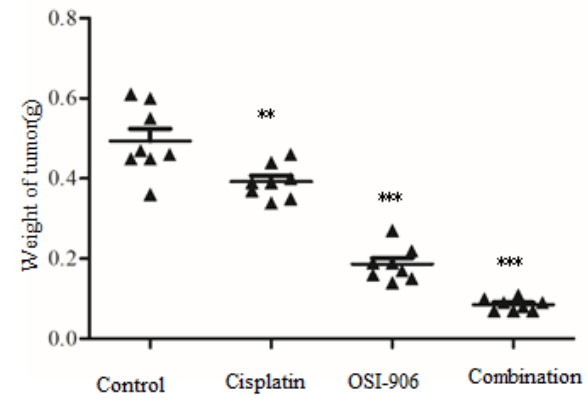

B

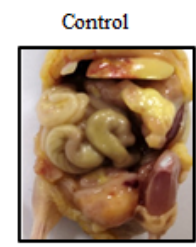

Cisplatin

OSI-906

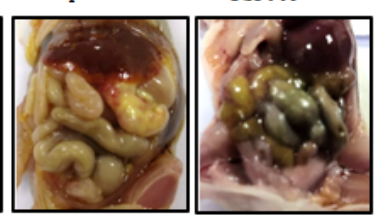

Combination
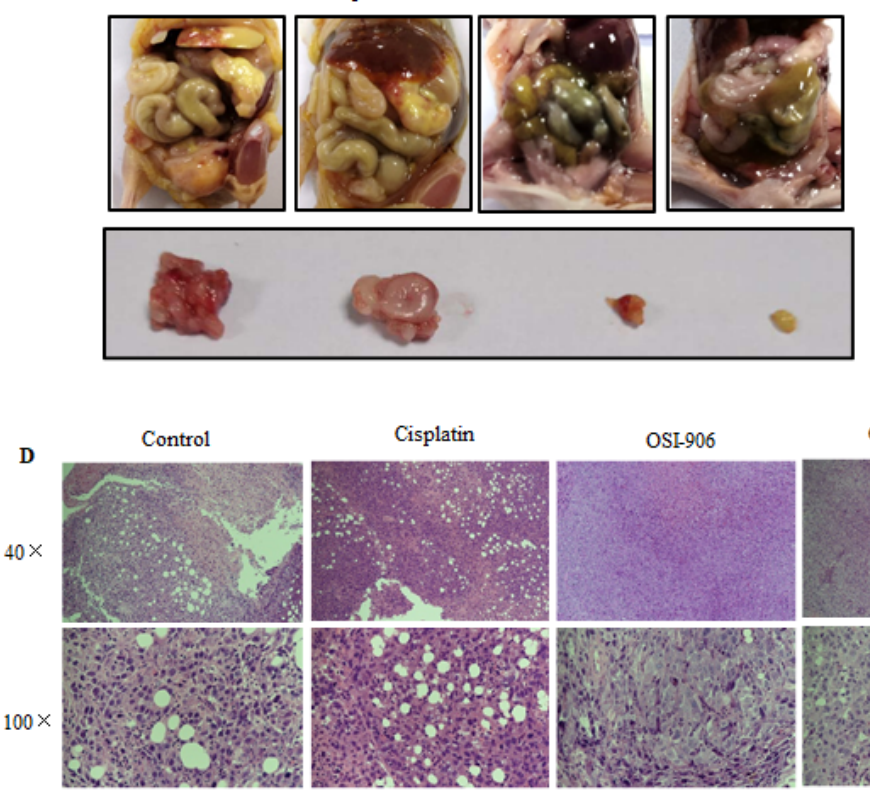

Cisplatin

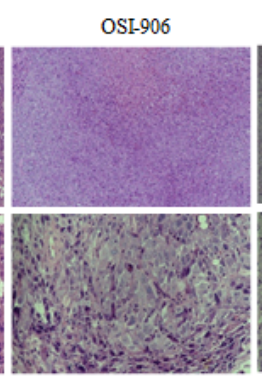

Combination

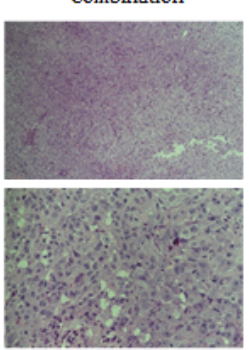

Figure 5 
OSI-906 restores the sensitivity of OCCC to cisplatin in vivo. (A) Animal experimental procedures. (B)Gross appearance of the abdominal cavities of the different groups. (C)Tumor weight was measured using an electronic balance. (D)HE staining was used to identify the tumor tissue of each group. ${ }^{* *} \mathrm{p} \rrbracket$ $0.01,{ }^{* * *} \mathrm{p} \otimes 0.001$.

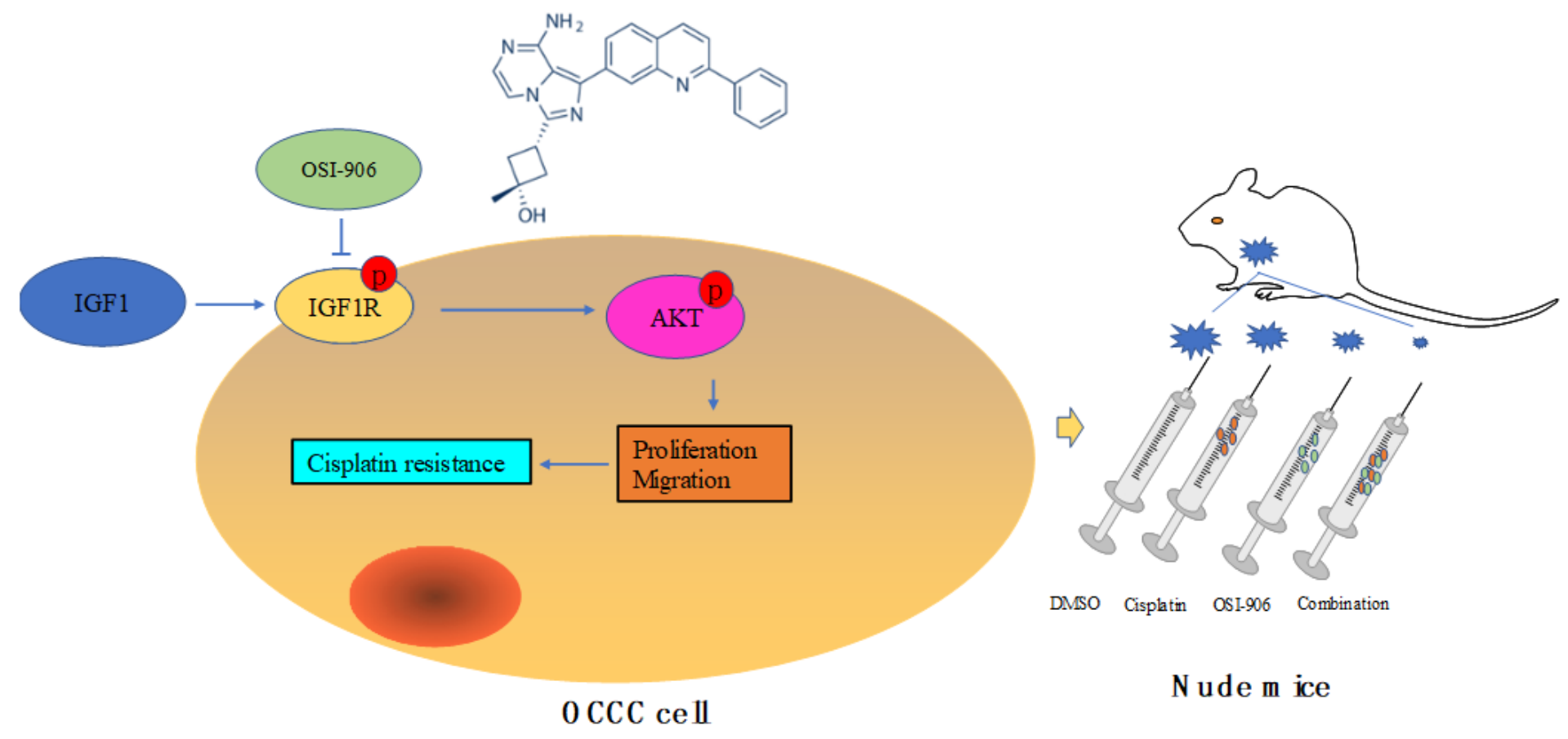

\section{Figure 6}

OSI-906 restores the sensitivity of OCCC to cisplatin in vivo. IGF1 is abnormally overexpressed in OCCC cells. IGF1 promotes proliferation and migration and induces cisplatin resistance in OCCC cells, while OSI906 reverses this effect. In vivo experiments also showed that OSI-906 increases sensitivity of OCCC to cisplatin. 